\title{
BIODYNAMIC CHARACTERISTICS OF VERTICAL AND DROP JUMPS
}

\author{
M. Čoh ${ }^{1}$, M. Bračič ${ }^{1}$, S. Peharec ${ }^{2}$, P. Bačić ${ }^{2}$, \\ M. Bratić ${ }^{3}$, M. Aleksandrović ${ }^{3}$ \\ ${ }^{1}$ Faculty of Sport, University of Ljubljana, Ljubljana, Slovenia \\ ${ }^{2}$ Peharec Polyclinic for Physical Medicine and Rehabilitation, \\ Pula, Croatia \\ ${ }^{3}$ Faculty of Sport and Physical Education, University of Niš, Serbia
}

\begin{abstract}
The aim of the study was to establish the main kinematic and dynamic parameters that generate the efficiency of vertical and drop jumps. The take-off power was assessed using the following tests: countermovement jump, counter-movement jump with arm swing, jump, drop jump and continuous jump. Kinematic and dynamic parameters of vertical and drop jumps were established using two separate forceplates Kistler Type 9286A and a synchronised 3-D kinematic system CCD SMART-600E. The athlete model was defined with 17 markers sensitive to infra-red light. It was established that the main generators of efficiency in vertical and drop jumps included: take-off velocity, eccentric-concentric time, eccentric impulse, ground contact time and ankle flexion.
\end{abstract}

Key words: diagnostics, kinematics, dynamics, vertical jump, drop jump.

\section{INTRODUCTION}

The movement structures that occur in specific sport situations are associated with different inputs of eccentric and concentric muscle contractions. The aim of training is often to modify the eccentric muscle contraction in view of its neurological characteristics. A good 
understanding of the role of eccentric muscle contraction in sport activities facilitates adaptation through the application of apposite training resources. The eccentric-concentric cycle consists of muscle stretching due to an external force and muscle shortening in the second phase, i.e. a stretch-shortening cycle (SSC) [12]. In the eccentric phase, a limited quantity of elastic energy accumulates in the muscle-tendon complex to be used in the second phase. This portion of elastic energy that is accumulated in the muscle is only available for a specific time. The available time depends on the life span of the cross-bridges and lasts from 15 to 120 milliseconds [8, 10, 14]. As regards the production of force, it is essential that the muscle develops more force and consumes less chemical energy during an eccentric contraction compared to a concentric contraction $[2,10,12,13]$. The efficiency of an eccentric-concentric contraction also depends on the time of the transition. The longer the time, the less efficient is the contraction. In addition to the extent and velocity of the change in the muscle's length and the duration of the transition, the efficiency of an eccentric-concentric contraction largely depends on pre-activation [9]. The latter defines the first contact of the foot with the ground. Preactivation prepares the muscles for stretching and is manifested in the number of attached cross-bridges and the change in the excitability of $\alpha$-motor nerves. Both factors affect the short-range stiffness of the muscle. Greater muscle stiffness causes a marked extension of the ligaments and the tendon which, in turn, reduces the consumption of chemical energy in the muscle. The reduced consumption of chemical energy is particularly important in those motor situations where specific movements must be made at a high velocity (e.g. the action of the ankle joint in a sprint, the take-off action in long jump, high jump and triple jump).

Even though the eccentric-concentric type of force production is very common in different human movements, it only became the focus of research relatively late. The first studies of this subject were conducted by Flitny and Hirst (1978) using an isolated muscle of a frog. The positive transfer of preliminary muscle stretching on the contractile characteristics of muscles in motor situations was first established by Asmussen and Bonde-Petersen [1] and Cavagna [8].

In functional and anatomical terms, the main role in a vertical jump is played by the two-joint thigh muscles which are also referred to as hamstrings or ischio-crural muscles by some authors [7, 19]. This muscle group includes: $\mathrm{m}$. semimembranosus, m. semitendinosus and 
$\mathrm{m}$. biceps femoris. In specific sport situations these muscles are responsible for the primary extension of the hip joint in a closed kinetic chain and for flexion of the knee joint. The length of the twojoint thigh muscle is highly variable, depending largely on the position of the knee and hip joints [18]. Its efficiency is best manifested in conditions of the high angular velocity of the joints of lower extremities. For that reason, the thigh muscles play an important role in fast explosive moves of acyclic and cyclic types.

Due to the inter-segmentary transmission of energy and the optimisation of the take-off action, the thigh muscles' activity is important in vertical jumps in conditions of a concentric or eccentricconcentric muscle contraction $[18,20]$. With vertical jumps, the muscles engage in the take-off action following the proximal-distal principle of muscle activation. In the first phase of the jump when the vertical velocity of the body's centre of gravity increases, the extensors of the trunk and hip are the most active muscles. The key role in this phase of the take-off action is played by m. gluteus maximus which is able to develop great force due to the relatively low angular velocity of the hips. The thigh muscles generate the peak activation at the beginning of the hip extension [7]. In the continuation of the take-off action, the knee extensors engage and the energy is transmitted from the hip to the knee through m. rectus femoris. The last phase of the take-off is performed by the two-joint shank muscle (m. gastrocnemius). The explosive performance of vertical jumps primarily depends on the optimal co-ordination of one- and two-joint muscles. Zajac [20] established that one-joint muscles generate the initial propulsive energy for vertical jumps, whereas two-joint muscles control the intermuscular co-ordination and the final vertical impulse. The method used for establishing the activation of muscles based on their electrical activity is electromyography (EMG).

In many sports, vertical and drop jumps are an important exercise resource in an athlete's power training. They enhance the eccentricconcentric muscle contraction of the lower extremities. At the same time, they are an indispensable measurement instrument in take-off power diagnostics. As regards their movement structure, vertical and drop jumps are similar to real motor situations in sports practice. Different test batteries are used to diagnose the explosive power of the lower extremities, either of a laboratory or a situational/field type. Bosco [5] developed a classical protocol to monitor take-off power based on vertical jumps. Take-off power in conditions where the 
active muscles first extend (eccentric contraction) and then shorten (concentric contraction) is measured by means of a countermovement vertical jump and a drop jump. Continuous jumps can be used as an instrument to measure reactive explosive power. This study aimed to, establish the main dynamic and kinematic parameters that generate the efficiency of vertical and drop jumps.

\section{METHODS}

\section{Experimental procedure}

In the experimental procedures, two elite athletes - triple jumpers took part (M. Š .: age 29 years, height $173.5 \mathrm{~cm}$, body mass $59.0 \mathrm{~kg}$; and M. G.: age 21 years, height $181.4 \mathrm{~cm}$, body mass $60.0 \mathrm{~kg}$ ) as well as two elite sprinters (M. O.: age 28 years, height $179.8 \mathrm{~cm}$, body mass $75.6 \mathrm{~kg}$; and J. G.: age 26 years, height $181.4 \mathrm{~cm}$, body mass $79.6 \mathrm{~kg}$ ). The athletes performed jumps in the following order: squat jump, countermovement jump, drop jump and continuous jumps. Each jump was performed three times. A system consisting of nine CCD SMART-e 600 video cameras (BTS Bioengineering, Padua) with a $50 \mathrm{~Hz}$ frequency and $768 \times 576$ pixel resolution was used for a 3-D kinematic analysis of vertical jumps (Figure 1). Kinematic parameters were processed by the BTS SMART Suite programme. A dynamic model featuring a system of 17 markers sensitive to infra-red light was defined (head, shoulders, forearm, upper arm, trunk, hips, thigh, shank, foot). The validity of the model was checked against the sequence of walking in the sagittal and frontal planes. The dynamic parameters of vertical and drop jumps were established by using two independent force plates (Kistler, Type 9286A). The sampling frequency was $1,000 \mathrm{~Hz}$. The analysis was based on the following dynamic parameters: peak ground reaction force, force impulse and amount of work per $1 \mathrm{~kg}$ of body mass (concentric work $\mathrm{J} / \mathrm{kg}$ ). The ground reaction force was measured uni- and bilaterally. The statistical analysis of the results was processed with the SPSS statistical software. 


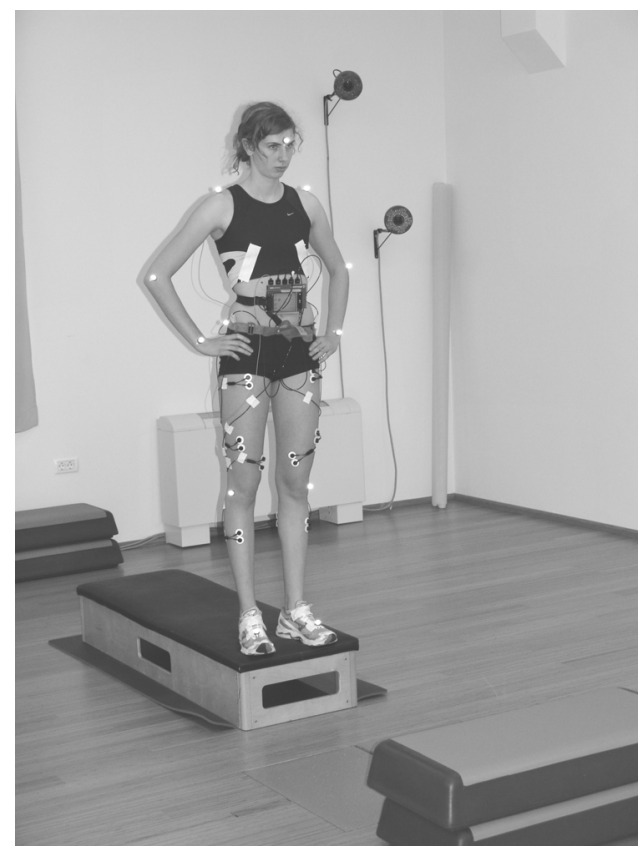

Figure 1. Drop jump $25 \mathrm{~cm}$ - kinematic and dynamic parameters

\section{RESULTS}

According to the standard test protocol [5] a vertical and drop jumps is initiated from a stationary upright posture, with the knee angle measuring about $90^{\circ}$. The jumps were performed with arms kept akimbo. The tests measured the eccentric - concentric component of speed strength. The measurement procedure was based on two separate Kistler force plates. The jumps height depends on the extensor muscles of the ankle, knee and hip joints. Based on the ground reaction force measured by force plates, and the 3D kinematic analysis the following kinematic and dynamic parameters were investigated (jump height, ground contact time, eccentric time, concentric time, flight time, jump efficiency, take-off velocity, peak force, eccentric-concentric impulse, hip fleksion, knee fleksion and ankle fleksion) (Table 1, 2, 3, 4). 
Table 1. Dynamic and kinematic parameters of a countermovement vertical jump

\begin{tabular}{|l|l|c|c|}
\hline COUNTERMOVEMENT JUMP & UNIT & $\mathrm{A}$ & $\mathrm{B}$ \\
\hline Jump height & $\mathrm{cm}$ & $48.8 \pm 0.3$ & $40.8 \pm 0.1$ \\
\hline Ground contact time & $\mathrm{ms}$ & $364 \pm 10$ & $338 \pm 9$ \\
\hline Eccentric time & $\mathrm{ms}$ & $123 \pm 9$ & $131 \pm 8$ \\
\hline Concentric time & $\mathrm{ms}$ & $241 \pm 6$ & $207 \pm 15$ \\
\hline Concentric work & $\mathrm{J} / \mathrm{kg}$ & $6.4 \pm 0.3$ & $5.1 \pm 0.1$ \\
\hline Jump efficiency & $\mathrm{cm} / \mathrm{J}$ & $7.6 \pm 0.4$ & $7.9 \pm 0.1$ \\
\hline Peak power & $\mathrm{W} / \mathrm{kg}$ & $50.1 \pm 0.9$ & $45.8 \pm 0.1$ \\
\hline Flight time & $\mathrm{ms}$ & $590 \pm 1$ & $525 \pm 1.9$ \\
\hline Take-off velocity & $\mathrm{m} / \mathrm{s}$ & $2.47 \pm 0.3$ & $2.42 \pm 0.3$ \\
\hline Peak force & $\mathrm{N}$ & $904 \pm 1.5$ & $1104 \pm 25$ \\
\hline Eccentric impulse & $\mathrm{Ns}$ & $34 \pm 1$ & $96 \pm 3$ \\
\hline Concentric impulse & $\mathrm{Ns}$ & $178 \pm 2$ & $145 \pm 1$ \\
\hline Hip flexion & $\mathrm{deg}$ & $77 \pm 1$ & $69 \pm 2$ \\
\hline Knee flexion & $\mathrm{deg}$ & $89 \pm 3$ & $88 \pm 2$ \\
\hline Ankle flexion & $\mathrm{deg}$ & $32 \pm 1$ & $38 \pm 2$ \\
\hline
\end{tabular}

Table 2. Dynamic and kinematic parameters of a countermovement vertical jump with an arm swing

\begin{tabular}{|l|l|c|c|}
\hline $\begin{array}{l}\text { COUNTERMOVEMENT } \\
\text { JUMP - WITH ARM SWING }\end{array}$ & UNIT & $\mathrm{A}$ & $\mathrm{B}$ \\
\hline Jump height & $\mathrm{cm}$ & $55.0 \pm 1.7$ & $47.3 \pm 0.5$ \\
\hline Ground contact time & $\mathrm{ms}$ & $372 \pm 11$ & $408 \pm 17$ \\
\hline Eccentric time & $\mathrm{ms}$ & $158 \pm 8$ & $128 \pm 8$ \\
\hline Concentric time & $\mathrm{ms}$ & $214 \pm 11$ & $280 \pm 7$ \\
\hline Concentric work & $\mathrm{J} / \mathrm{kg}$ & $6.4 \pm 0.3$ & $5.8 \pm 0.1$ \\
\hline Jump efficiency & $\mathrm{cm} / \mathrm{J}$ & $8.6 \pm 0.2$ & $8.1 \pm 0.1$ \\
\hline Peak power & $\mathrm{W} / \mathrm{kg}$ & $57.3 \pm 2.2$ & $49.3 \pm 0.6$ \\
\hline Flight time & $\mathrm{ms}$ & $633 \pm 9$ & $578 \pm 5$ \\
\hline Take-off velocity & $\mathrm{m} / \mathrm{s}$ & $2.80 \pm 0.4$ & $2.68 \pm 0.3$ \\
\hline Peak force & $\mathrm{N}$ & $1029 \pm 23$ & $827 \pm 6$ \\
\hline Eccentric impulse & $\mathrm{Ns}$ & $93 \pm 3$ & $39 \pm 4$ \\
\hline Concentric impulse & $\mathrm{Ns}$ & $181 \pm 5$ & $174 \pm 1$ \\
\hline Hip flexion & $\mathrm{deg}$ & $74 \pm 3$ & $70 \pm 2$ \\
\hline Knee flexion & $\mathrm{deg}$ & $88 \pm 2$ & $96 \pm 1$ \\
\hline Ankle flexion & $\mathrm{deg}$ & $31 \pm 1$ & $40 \pm 1$ \\
\hline
\end{tabular}


Table 3. Dynamic and kinematic parameters of a drop jump from a height of $25 \mathrm{~cm}$

\begin{tabular}{|l|l|c|c|}
\hline DROP JUMP $-\mathbf{2 5} \mathbf{~ c m}$ & UNIT & A & B \\
\hline Jump height & $\mathrm{cm}$ & $47.3 \pm 0.8$ & $38.6 \pm 0.8$ \\
\hline Ground contact time & $\mathrm{ms}$ & $165 \pm 5$ & $174 \pm 3$ \\
\hline Eccentric time & $\mathrm{ms}$ & $73 \pm 2$ & $80 \pm 2$ \\
\hline Concentric time & $\mathrm{ms}$ & $92 \pm 2$ & $94 \pm 1$ \\
\hline Concentric work & $\mathrm{J} / \mathrm{kg}$ & $5.1 \pm 0.2$ & $4.3 \pm 0.1$ \\
\hline Jump efficiency & $\mathrm{cm} / \mathrm{J}$ & $9.3 \pm 0.3$ & $9.0 \pm 0.3$ \\
\hline Peak power & $\mathrm{W} / \mathrm{kg}$ & $92.4 \pm 6.7$ & $76.6 \pm 1.2$ \\
\hline Flight time & $\mathrm{ms}$ & $580 \pm 3$ & $503 \pm 6$ \\
\hline Take-off velocity & $\mathrm{m} / \mathrm{s}$ & $2.78 \pm 0.1$ & $2.65 \pm 0.4$ \\
\hline Peak force & $\mathrm{N}$ & $3654 \pm 108$ & $3689 \pm 180$ \\
\hline Eccentric impulse & $\mathrm{Ns}$ & $161 \pm 4$ & $151 \pm 3$ \\
\hline Concentric impulse & $\mathrm{Ns}$ & $164 \pm 1$ & $141 \pm 2$ \\
\hline Hip flexion & $\mathrm{deg}$ & $26 \pm 1$ & $29 \pm 1$ \\
\hline Knee flexion & $\mathrm{deg}$ & $56 \pm 2$ & $61 \pm 2$ \\
\hline Ankle flexion & $\mathrm{deg}$ & $13 \pm 1$ & $25 \pm 1$ \\
\hline
\end{tabular}

Table 4. Dynamic and kinematic parameters of continuous jumps $(n=20)$

\begin{tabular}{|l|l|c|c|}
\hline CONTINUOUS JUMPS $(\mathbf{N}=\mathbf{2 0})$ & UNIT & $\mathrm{C}$ & $\mathrm{D}$ \\
\hline Jump height & $\mathrm{cm}$ & $33.3 \pm 1.5$ & $37.5 \pm 3.8$ \\
\hline Ground contact time & $\mathrm{ms}$ & $156 \pm 4$ & $252 \pm 19$ \\
\hline Eccentric time & $\mathrm{ms}$ & $79 \pm 6$ & $128 \pm 8$ \\
\hline Concentric time & $\mathrm{ms}$ & $76 \pm 3$ & $124 \pm 11$ \\
\hline Concentric work & $\mathrm{J} / \mathrm{kg}$ & $3.1 \pm 0.2$ & $4.0 \pm 0.4$ \\
\hline Jump efficiency & $\mathrm{cm} / \mathrm{J}$ & $10.8 \pm 0.5$ & $9.4 \pm 0.2$ \\
\hline Peak power & $\mathrm{W} / \mathrm{kg}$ & $66.3 \pm 4.4$ & $58.8 \pm 7.1$ \\
\hline Flight time & $\mathrm{ms}$ & $445 \pm 13$ & $452 \pm 34$ \\
\hline Index activity & & $0.35 \pm 0.2$ & $0.55 \pm 0.4$ \\
\hline Take-off velocity & $\mathrm{m} / \mathrm{s}$ & $2.19 \pm 0.7$ & $2.59 \pm 0.2$ \\
\hline Peak force & $\mathrm{N}$ & $4224 \pm 393$ & $2735 \pm 363$ \\
\hline Eccentric impulse & $\mathrm{Ns}$ & $165 \pm 17$ & $171 \pm 23$ \\
\hline Concentric impulse & $\mathrm{Ns}$ & $155 \pm 16$ & $201 \pm 26$ \\
\hline Hip flexion & $\mathrm{deg}$ & $12 \pm 3$ & $22 \pm 6$ \\
\hline Knee flexion & $\mathrm{deg}$ & $27 \pm 3$ & $46 \pm 4$ \\
\hline Ankle flexion & $\mathrm{deg}$ & $6 \pm 2$ & $18 \pm 2$ \\
\hline
\end{tabular}




\section{DISCUSSION}

This motor task consists of a rapid lowering of the body's centre of gravity, whereby the active leg muscles stretch (eccentric contraction). Then the movement is halted and the subject immediately takes off in a vertical direction (concentric contraction). The energy that accumulates in the muscles and the tendon during the stretching is transmitted to the concentric phase. Consequently, the velocity of movement in the second phase increases. The jump is performed with arms kept akimbo. The measurement protocol was based on the following parameters (Table 1). The take-off time ranges from 338 to 364 milliseconds. According to the principle of elastic energy transfer, the eccentric phase is shorter than the concentric one. Subject A, whose jump height was $48.8 \mathrm{~cm}$, had an eccentric/concentric phase ratio of $33.8 \%: 66.2 \%$. For subject B, whose jump height was $40.8 \mathrm{~cm}$, the respective ratio was $38.7 \%: 61.3 \%$. It can be concluded that the efficiency of the two-phase muscular activity depends on an appropriate ratio between the eccentric and concentric muscle contraction. The elastic energy generated in the first phase thus accumulates in the muscle-tendon complex (tendon, aponeurosis, cross-bridges, perimysium, epimysium and sarcolemmas; $[3,4])$ and merges with the chemical energy of the muscle in the concentric phase. The consequence is a larger muscle force, resulting in a higher jump. The vertical velocity of the body's centre of gravity in a twophase muscle contraction is $9.5 \%$ higher than in a single-phase concentric muscle contraction. The transition from eccentric to concentric contraction takes place when the knee angle measures $89^{\circ}$. The ground reaction force ranges between $904 \mathrm{~N}$ and $1104 \mathrm{~N}$.

A countermovement vertical jump can be performed with or without an arm swing. According to the classical protocol [5], the arms were isolated and kept akimbo. In some motor situations it is important to know what the contribution of the arms is to the efficiency of a vertical jump (e.g. in volleyball). The arms contribute $8.6 \%$ to $8.8 \%$ to the jump height (Table 2). The arm swing changes some kinematic and dynamic parameters of a vertical jump. For study subject A, the ground contact time was longer, especially the eccentric phase, while increases were recorded in the ground reaction force - by $125 \mathrm{~N}$, take-off velocity - by $0.33 \mathrm{~m} / \mathrm{s}$ and the force impulse in the eccentric contraction - by 59 Ns. 
Drop jumps are an extremely efficient means to improve how the ankle muscles function in eccentric-concentric conditions. At the same time, they are used in take-off power diagnostics. The degree of loading in drop jumps is defined by the jump depth, the athlete's body mass, contact time and vertical jump height. The jumps are performed from a height of $25 \mathrm{~cm}$ to $80 \mathrm{~cm}$. Jumps from a height of $80 \mathrm{~cm}$ or more constitute a risk of injury which is why they are usually warned against in training practice. These jumps put the bulk of loading on the plantarflexors and the knee and hip extensors. The landing must be performed in such a way as to prevent the heel from touching the ground. The bumping of the heel against the ground causes a rise in the ground reaction force of more than $100 \%$. If braking in the eccentric phase cannot be performed by the ankle joint extensors, the task is taken over by the knee and hip extensors. However, in this case, the time of the transition from the eccentric to the concentric phase is substantially longer, thus negatively affecting the efficiency of the jump.

A drop jump consists of the following phases: leaving the bench set at a specific height, flight, preparation for landing, ground contact, braking, eccentric-concentric contraction and vertical acceleration. Not only is the height of the bench important, but so too is the peak height of the body's centre of gravity during the flight phase. The preactivation phase starts 100 milliseconds before the ground contact [9, 11]. The function of the muscle pre-activation is to prepare the muscle for stretching. This pre-activation is secured by the concurrent activation of $\mathrm{m}$. gastrocnemius and $\mathrm{m}$. tibialis anterior. The shortrange stiffness of $\mathrm{m}$. gastrocnemius thus facilitates the accumulation of a higher quantity of elastic energy in the tendon and a smaller extension of the muscle [9]. The purpose of drop jumps is to shorten the time of shock absorption which generates an optimum transition from the eccentric to the concentric contraction. The analysis of drop jumps was based on the following parameters (Table 3 ).

Contact time is one of the crucial parameters of drop jumps as it defines the efficiency of the transition from the eccentric to the concentric contraction. The contact time of the study subjects who performed a drop jump from a height of $25 \mathrm{~cm}$ ranged from 165 to 174 milliseconds. The ratio between the time of the eccentric and concentric phases of subject A was $44.2 \%$ : $55.8 \%$, whereas that for subject B was $45.9 \%: 54.1 \%$. Subject A expressed greater efficiency in braking after landing from a 47.3 centimetre-high jump. Some 
authors $[11,19]$ have shown that the pre-activation of plantarflexors is the most important mechanism in shortening the contact time in eccentric-concentric motor tasks. Basically, the central motor programme improves and - through control of musculature activation - increases the short-range stiffness of the muscle-tendon complex of the lower extremities upon contact with the ground. This programme is responsible for synchronisation of the ankle joint flexors and extensors. The optimum pre-activation of agonists and antagonists prior to contacting the ground reduces the amplitudes of movement and shortens the eccentric phase. Consequently, a larger muscle force is generated, along with a lower consumption of metabolic muscle energy. The shortening of the ground contact time in a drop jump is mainly the result of the pre-activation of $\mathrm{m}$. gastrocnemius, $\mathrm{m}$. soleus and $\mathrm{m}$. tibialis anterior [11]. The efficiency of the performance of drop jumps in subject $A$ (this athlete is an elite triple jumper) compared to that in subject $\mathrm{B}$ is primarily reflected in the following parameters: a shorter contact time, a shorter eccentric phase, peak power $(\mathrm{W} / \mathrm{kg}$ of body mass), a higher take-off velocity, a higher force impulse in the eccentric and particularly the concentric phase as well as a lower amplitude of movement in the knee and ankle joints. Short-range muscle stiffness is additionally manifested in the ankle joint since the amplitude of the movement of subject A was only $13^{\circ}$, whereas that of subject $\mathrm{B}$ was $25^{\circ}$. The peak ground reaction force, measured by two force plates (Kistler, Type 9286A), was $3654 \pm 108 \mathrm{~N}$ in the case of subject $\mathrm{A}$ and $3689 \pm 180 \mathrm{~N}$ in the case of subject B (drop jump from $25 \mathrm{~cm}$ ). The peak ground reaction force and the peak force impulse were recorded in the eccentric phase.

The test consists of a series of continuous vertical jumps (Table 4), with the emphasis put on the take-off from the ankle joint. The arms are kept akimbo, while attention must be paid to a correct body balance. The subjects of the present study performed 20 vertical jumps. The test assessed the degree of the elastic (reactive) power of the plantarflexors which play the key role in real motor situations such as sprint, hurdles, long jump and triple jump. The test result is defined by the average height of the jumps, the average time of flight and the ground contact phases, the average duration of eccentric and concentric phases, the peak ground reaction force and the realised average power per $1 \mathrm{~kg}$ of the subject's body mass (W/kg).

Study subject $\mathrm{C}$, who is an elite world class sprinter, recorded very short contact times, averaging $156 \pm 4$ milliseconds. The eccentric 
phase lasted only 79 milliseconds. The velocity of the eccentricconcentric cycle reveals the efficiency of the transformation of kinetic energy to elastic energy, with major roles being played by the elastic and reflex components of the muscle-tendon complex. The shorter the phase during which the muscle-tendon complex stretches and the smaller the amplitude of movement in the ankle $\left(6^{\circ}\right)$, the better the utilisation of elastic energy $[6,15]$. In terms of the movement structure and generation of force within a short time interval, the test is very similar to that for sprinting. Sprinting is a typical example of an eccentric-concentric cycle. In the eccentric contact phase, the muscle-tendon complex extends, whereby the accumulated elastic energy is consumed in the efficient propulsion of the running stride. In the phase of peak sprint velocity, the contact phase time ranges between 85 and 90 milliseconds, with the vertical component of the ground reaction force equalling 1400 to $2100 \mathrm{~N}$ [14]. Subject C develops a peak ground reaction force of $2265 \pm 373 \mathrm{~N}$ and $1959 \pm$ $413 \mathrm{~N}$ with their right and left legs, respectively. A certain degree of asymmetry between the lower extremities was identified. The obvious difference between study subjects $\mathrm{C}$ and $\mathrm{D}$ was seen in the short-range stiffness of their ankle and knee joints. Subject C's flexion of the ankle joint in vertical jumps was only $6 \pm 2^{\circ}$, whereas that of subject $\mathrm{D}$ was $18 \pm 2^{\circ}$. The greater short-range stiffness of the ankle joint in a sprint results in the lower consumption of chemical energy in the following muscles: $\mathrm{m}$. gastrocnemius lateralis, $\mathrm{m}$. gastrocnemius medialis and $\mathrm{m}$. soleus. The lower consumption of chemical energy is important in those motor situations where a certain movement must be performed at a high velocity.

\section{CONCLUSION}

The biomechanical diagnostics of the explosive power of lower extremities is an extremely important element of monitoring modern athletes' training processes. The results of measuring different types of vertical and drop jumps provide us with fundamental information on the status and functioning of the neuromuscular system. Based on this information the training process can be far more accurately programmed and controlled in terms of power. Certain state-of-the-art technologies and measurement procedures for diagnosing explosive power have been presented, primarily as regards monitoring the 
dynamic and kinematic parameters of vertical and drop jumps. The tests are implemented in laboratory conditions, whereas the next step is to enable the monitoring of an athlete's neuromuscular system in field conditions, i.e. in a real environment.

\section{REFERENCES}

1. Asmussen E., Bonde-Petersen F. (1974) Storage of elastic energy in skeletal muscles in man. Acta Physiol. Scand. 91: 385-392

2. Bührle M., Schmidtbleicher D., Ressel H. (1983) Die spezielle diagnose der einzelnen kraftkomponenten in hochleistungssport. Leistungssport. 3: 11-16

3. Bobbert M., Huijing P., van Ingen Schenau G. (1987) Drop jumping I. The influence of jumping technique on the biomechanics of jumping. Med. Sci. Sport Exerc. 19: 332-338

4. Bobbert M., van Ingen Schenau G. (1988) Coordination in vertical jumping. J. Biomech. 21: 249-262

5. Bosco C. (1992) L'evaluation de la force par le test de Bosco. Roma, Societa Stampa Sportiva

6. Bosco C., Vittori C., Matteuci E. (1995) Considerazioni sulle variazioni dinamiche di alcuni parametri biomeccanici nela corsa. Atleticastudi. 2: 155-162

7. Brockett C., Morgan D., Proske U. (2004) Predicting hamstring strain injury in elite athletes. Med. Sci. Sports Exerc. 36: 379-387

8. Cavagna G. (1977) Storage and utilization of elastic energy in skeletal muscle. Exerc. Sport Sci. Rev. 5: 89-129

9. Dolenec A. (1999) Vpliv treniranja globinskih skokov s plantarno in dorsalno tehniko na delo gležnja pri globinskih skokih [Impact of training of drop jumps using plantar and dorsal technique on the work of ankle in drop jumps]. Doctoral dissertation, Faculty of Sport, Ljubljana

10. Enoka R. (2003) Neuromechanics of human movement. Human Kinetics, Champaign, IL

11. Gollhofer A., Kyrolainen H. (1991) Neuromuscular control of the human leg extensor muscles in jump exercises under various stretchload conditions. Int. J. Sports Med. 12: 34-40

12. Komi P., Gollhofer A. (1997) Stretch reflex can have an important role in force enhancement during SSC exercises. J. Appl. Biomech. 13: 451-459

13. Mero A., Komi P. (1994) EMG, force, and power analysis of sprintspecific strength exercises. J. Appl. Biomech. 10: 1-13 
14. Mero A., Komi P., Gregor R. (1992) Biomechanics of sprint running. Sport Med. 13: 376-392

15. Mero A., Kuitunen S., Harland M., Kyrolainen H., Komi P. (2006) Effects of muscle-tendon length on joint movement and power during sprint starts. J. Sport Sci. 24: 165-173

16. Markovic G., Dizdar D., Jukic I., Cardinale M. (2004) Reliability and factorial validity of squat and countermovement jump tests. J. Strength Con. Res. 16: 20-31

17. Newton R., Kraemer W. (1994) Developing explosive muscular power: implications for a mixed methods training strategy. J. Strength Cond. Res. 18: 551-555

18. Šarabon N., Fajon M., Zupanc O., Draksler J. (2005) Stegenske strune [Hamstrings injuries]. Šport. 3: 45-52

19. Schmidtbleicher D. (1992) Training for power sports. In: Strength and power sport, P. Komi (ed). Blackwell Scientific, London. 381395

20. Zajac F. (1993) Muscle coordination of movement: a perspective. J. Biomech. 26: 109-124

\section{Correspondence to:}

Milan Coh

University of Ljubljana

Ljubljana

Slovenia

E-mail: Milan.coh@fsp.uni-lj.sl 